\title{
COMMENTARY
}

\section{A dynamic view of mHLA-DR expression in management of severe septic patients}

\author{
Guillaume Monneret ${ }^{1,2 *}$, Alain Lepape ${ }^{2,3}$ and Fabienne Venet ${ }^{1,2}$ \\ See related research by Wu et al., http://ccforum.com/content/15/5/R220
}

\begin{abstract}
Increasing evidence suggests that the secondary phase of sepsis (that is, after the first proinflammatory hours) is characterized by the occurrence of a systemic failure of the immune system. In the most immunodepressed patients, therapies could be used to restore normal immune functions. However, biomarkers need to be developed to beforehand specifically identify these patients. Of these biomarkers, diminished monocyte HLA-DR expression has rapidly become the most popular. Herein, novel perspectives regarding monocyte HLA-DR assessed as a dynamic parameter in septic patients will be discussed in the context of a recently published study investigating daily evolution of monocyte HLA-DR with regard to 28 day-mortality after severe sepsis.
\end{abstract}

The work of Wu and colleagues [1] is in accordance with the recent concept of sepsis-induced immunosuppression $[2,3]$. There is now agreement that many severe septic patients survive the first critical hours of the syndrome but eventually die later in a state of immunosuppression that is illustrated by patients' difficulty to fight the primary bacterial infection, decreased resistance to secondary nosocomial infections and reactivation of viral infections normally solely pathogenic in the immunocompromised host $[4,5]$. Consequently, immunostimulatory therapies might be used to restore immune functions in the most immunodepressed patients [6]. In the absence of any specific clinical signs of immune failure, however, it is beforehand critical to determine the best biological tools (markers of septic patients' immune failure) enabling patient stratification [7].

*Correspondence: guillaume.monneret@chu-lyon.fr

${ }^{1}$ Hospices Civils de Lyon, Immunology Laboratory, Hôpital E. Herriot, 5 place

d'Arsonval, 69437 Lyon cedex 03, France

Full list of author information is available at the end of the article
The most frequently assessed biomarker in the field to date is undeniably the measurement of HLA-DR expression on circulating monocytes (mHLA-DR). There appears to be general consensus that diminished mHLA-DR is a reliable marker for the development of immunosuppression in critically ill patients. Indeed, decreased expression of this marker is regularly reported to be associated with higher mortality/risk for nosocomial infections in critically ill patients. More than 100 articles on this topic have been published in different ICU conditions, including sepsis, trauma, burns, and stroke.

It is becoming increasingly clear that the critical point after injury is the recovery of normal mHLA-DR. Schematically, mHLA-DR rapidly returns to normal values (generally in less than 1 week) in injured patients with uneventful recovery, whereas this parameter remains constantly decreased in patients with adverse outcome/ secondary septic complications. In line with this hypothesis, Wu and colleagues showed that low mHLADR was associated with increased mortality in severe sepsis [1]. Most importantly, the authors propose that, more than a single value at a given time point, the dynamic change of mHLA-DR over time would be a better predictor of mortality. Indeed, in their study, single measurements of mHLA-DR within the first week after patient admission (either days 0,3 or 7 ) had no predictive value regarding mortality. In contrast, results expressed as dynamic parameters (that is, $\Delta$ between two time points) provide excellent predictive values, especially $\Delta$ calculated between days 0 and 3 or between days 0 and 7 (areas under the curve of 0.92 and 0.94 , respectively, in receiver operating characteristic analysis). Most importantly, after multivariate analysis, the authors show that these two parameters remain the sole independent predictors of mortality with an elevated significant odds ratio.

Overall, the present results confirm the concept that patients who do not start to restore normal immune functions are those who are going to die [1]. These results are in agreement with two recent studies in which a weak slope of mHLA-DR recovery was associated with increased risk of secondary infections in a mixed ICU 
population [8] and in trauma [9]. This outcome could have important consequences in patient management, by potentially allowing for the administration of tailored therapies aimed at restoring immune functions based on dynamic changes of immunological parameters.

There are some limitations to $\mathrm{Wu}$ and colleagues' study that should be noted. First, the study is monocentric in a small cohort of surgical patients (that is, not necessarily representative of the whole septic population) that present with relatively elevated mHLA-DR values $(>50 \%)$ in comparison with results from the literature (usually below $50 \%$ in severe septic patients). This moderate severity and the lack of statistical power due to the small size of the cohort may explain surprising results after multivariate analysis (Sequential Organ Failure Assessment and Acute Physiology and Chronic Health Evaluation II scores were not significantly different between survivors and nonsurvivors, odds ratio with very large confidence intervals). Another limitation lies in the expression of mHLA-DR results. Indeed, the standardized recommended method for expressing mHLA-DR results is as numbers of antibodies bound per cell and not as the percentage of positive cells $[10,11]$. As an example, the authors suggest that a difference of $4.8 \%$ in mHLA-DR between days 0 and 3 is of significance. Although most probably correct from a statistical perspective, this threshold is hardly applicable in routine/technical practice because such a small percentage difference could be due to measurement variability by flow cytometry.

Overall and beyond these limitations, appropriately acknowledged by the authors, this study confirms that after injury (for example, severe sepsis) survivors tend to progressively normalize mHLA-DR, contrary to nonsurvivors. This biologic parameter could thus provide critical information when assessed as a dynamic variable over time. This potential aspect now deserves to be validated in multicentric clinical studies using standardized flow cytometry protocols.

Abbreviations

mHLA-DR, monocyte human leukocyte antigen-DR.

Competing interests

The authors declare that they have no competing interests.

\section{Author details}

${ }^{1}$ Hospices Civils de Lyon, Immunology Laboratory, Hôpital E. Herriot, 5 place d'Arsonval, 69437 Lyon cedex 03, France. 'Equipe d'Accueil Mixte 4174, Hémostase, Inflammation, Sepsis, Hospices Civils de Lyon, Université Claude Bernard Lyon 1, Faculté de Médecine Laennec, 8 rue Guillaume Paradin, 69372 Lyon cedex 08, France. ${ }^{3}$ Hospices Civils de Lyon, Intensive Care Unit, Lyon-Sud University Hospital, 165 chemin du grand Revoyet, 69495 Pierre-Bénite cedex, France.

Published: 17 October 2011

\section{References}

1. Wu J-F, Jie Ma JC, Ou-Yang B, Chen M-Y, Li LF, Liu Y-J, Lin A-h, Guan X-D: Changes of monocyte human leukocyte antigen-DR expression as a reliable predictor of mortality in severe sepsis. Crit Care 2011, 15:R220.

2. Hotchkiss RS, Karl IE: The pathophysiology and treatment of sepsis. N Engl J Med 2003, 348:138-150

3. Monneret $\mathrm{G}$, Venet $\mathrm{F}$, Pachot $\mathrm{A}$, Lepape A: Monitoring immune dysfunctions in the septic patient: a new skin for the old ceremony. Mo/ Med 2008, 14:64-78.

4. Monneret G, Venet F, Kullberg BJ, Netea MG: ICU-acquired immunosuppression and the risk for secondary fungal infections. Med Mycol 2011, 49(Suppl 1):S17-S23.

5. Kalil AC, Florescu DF: Prevalence and mortality associated with cytomegalovirus infection in nonimmunosuppressed patients in the intensive care unit. Crit Care Med 2009, 37:2350-2358.

6. Pugin J: Immunostimulation is a rational therapeutic strategy in sepsis. Novartis Found Symp 2007, 280:21-27; discussion 27-36, 160-164.

7. Meisel C, Schefold JC, Pschowski R, Baumann T, Hetzger K, Gregor J, WeberCarstens S, Hasper D, Keh D, Zuckermann H, Reinke P, Volk HD: GM-CSF to reverse sepsis-associated immunosuppression: a double-blind randomized placebo-controlled multicenter trial. Am J Respir Crit Care Med 2009, 180:640-648.

8. Lukaszewicz AC, Grienay M, Resche-Rigon M, Pirracchio R, Faivre V, Boval B, Payen D: Monocytic HLA-DR expression in intensive care patients: interest for prognosis and secondary infection prediction. Crit Care Med 2009, 37:2746-2752

9. Cheron A, Floccard B, Allaouchiche B, Guignant C, Poitevin F, Malcus C, Crozon J, Faure A, Guillaume C, Marcotte G, Vulliez A, Monneuse O, Monneret G: Lack of recovery in monocyte human leukocyte antigen-DR expression is independently associated with the development of sepsis after major trauma. Crit Care 2010, 14:R208.

10. Docke WD, Hoflich C, Davis KA, Rottgers K, Meisel C, Kiefer P, Weber SU, Hedwig-Geissing M, Kreuzfelder E, Tschentscher P, Nebe T, Engel A, Monneret G, Spittler A, Schmolke K, Reinke P, Volk HD, Kunz D: Monitoring temporary immunodepression by flow cytometric measurement of monocytic HLA-DR expression: a multicenter standardized study. Clin Chem 2005, 51:2341-2347

11. Venet F, Lepape A, Monneret $\mathrm{G}$ : Flow cytometry perspectives in the ICU: from diagnosis of infection to monitoring of injury-induced immune dysfunctions. Crit Care 2011, 15:231.

doi:10.1186/cc10452

Cite this article as: Monneret $G$, et al: A dynamic view of mHLA-DR expression in management of severe septic patients. Critical Care 2011, 15:198. 